\title{
HISTOIRE ALLEMANDE ET TOTALITÉ : LEOPOLD VON RANKE, JOHANN GUSTAV DROYSEN, KARL LAMPRECHT *
}

Les écrits de Leopold von Ranke, Johann Gustav Droysen et Karl Lamprecht sont le lieu de l'émergence des concepts principaux de l'historiographie allemande au XIX' siècle. En deçà de l'opposition entre l'historisme des deux premiers auteurs et la Kulturgeschichte du dernier, il apparaît que le problème de la totalité, commun aux trois, est essentiel. On assiste, en effet, à la revendication de l'autonomie de l'histoire, mais comme synthèse des autres savoirs, et de sa scientificité par la mise en ordre des phénomènes en une unité spatiale et une continuité temporelle.

L'individualisme méthodologique de l'historisme fait place à l'histoire comme savoir nomothétique : avec Lamprecht, l'histoire " nouvelle ", générique, causale, des " processus légaux » ou " situations " (Zustandgeschichte) s'oppose à l'ancienne histoire " individualiste ", téléologique des " événements " et des personnalités éminentes. La totalité prend différents noms pour caractériser l'activité de l'historien ou la structure de l'objet. La notion romantique d'" organisme ", l'image du cercle, se prolongent dans les notions de "connexion" (Zusammenhang) et "système " chez Ranke, de "forme " (Gestalt) totalisante chez Droysen, et de « situation " (Zustand) chez Lamprecht. Il y a passage

\footnotetext{
* Des éléments de ce texte ont fait l'objet d'un débat interdisciplinaire au séminaire Questions d'histoire de l'anthropologie, animé par $\mathrm{M}^{\mathrm{me}}$ Rupp-Eisenreich à l'E.H.E.S.S., le 7 janvier 1987. Pour une étude complexe, on consultera : Catherine DEvULDER, L'Histoire en Allemagne au XIX' siècle : méthode et pensée historiques chez Leopold von Ranke, Johann Gustav Droysen, et Karl Lamprecht, Thèse de doctorat d'État, Strasbourg II, 1987. Les traductions sont de l'auteur.
}

Revue de synthese : IV $\mathrm{S}^{\mathrm{N}} \mathrm{N}^{\circ} 2$, avril-juin 1987. 
d'une totalité spirituelle à une totalité empirique. L'objet historique dans l'historisme fait partie d'une totalité qui le dépasse - l'esprit - et aspire à l'absolu divin. Chez Lamprecht, l'objet se concrétise et regroupe, pour ainsi dire, tous les niveaux de l'activité humaine.

La totalité se retrouve tant au niveau logique (le processus de la connaissance est un système pour l'historisme) qu'au niveau idéologique (l'historien a affaire à un objet global et complexe, l'État-nation dont l'équilibre repose sur le consensus gentium) et tant au niveau du rapport de l'historien à son objet (soit le cercle herméneutique pour l'historisme) qu'au niveau de l'objet lui-même (ce dernier est structuré).

L'histoire se pose en s'opposant aux savoirs contemporains et se présente comme étant au sommet d'une hiérarchie des connaissances en ayant opéré leur synthèse. Ainsi, chez Ranke, elle est l'accomplissement de la philosophie et de l'art en ayant pour spécificité le champ du réel. Chez Droysen, l'histoire, recherchant le changement dans le semblable, s'oppose aux sciences de la nature qui conçoivent ce qui est semblable dans le changement, mais logique, physique et éthique ou histoire ne sont que les trois côtés d'un prisme où se reflète la lumière éternelle. Chez Lamprecht, l'histoire positiviste aspire à une unité des sciences, mais n'est qu'une projection des catégories de la psychophysiologie de Wundt, en une psychologie évolutionniste.

\section{L'HISTORISME ET LE CERCLE HERMÉNEUTIQUE}

L'historisme prônant l'histoire comme connaissance applique le schéma de l'optique. La totalisation de la réalité historique est supposée par la circularité du savoir historique entre le passé et le présent : l'histoire est une image rendue présente, temporalisée par la position de l'historien. Ceci suppose un accord entre le sujet et l'objet : le but de Ranke est "l'identification de la réflexion avec les faits " ', " ce qui se passe et la science [de ce qui se passe] doivent coïncider entièrement " ${ }^{2}$. Cette coïncidence est * la concordance des lois d'après lesquelles [...] l'esprit considérant procède, avec celles par lesquelles l'esprit considéré fait saillie " ${ }^{3}$. La science doit s'avancer vers l'objet et, ou bien l'assimiler, ou bien le laisser être : «c'est que la vision subjective doit s'éclipser

1. Volker DotTerweich, Walther Peter Fuchs, eds, Leopold von Ranke. Vorlesungseinleitungen, Munich/Vienne, Oldenburg, 1975, p. 142 (cité par la suite comme DOTTERWEICH, FuCHS).

2. Ibid., p. 188.

3. Ibid., p. 78. 
complètement sous la vérité objective " ${ }^{4}$, ou : « la science prend en elle l'objet " ${ }^{5}$. Mais l'objet devient objet de science, est objectif, parce que, par lui-même, il signifie.

Droysen insiste plus sur le cercle herméneutique : la compréhension du passé par l'historien est permise par l'interaction entre le passé et le présent, et la position de l'historien dans le cercle du devenir. Elle prend modèle sur l'intersubjectivité. L'objet historique est conforme à l'être humain ; réfléchir sur l'histoire pour Droysen, c'est réfléchir sur le moi de la personnalité. La totalité donne à l'homme sa forme et son identité : non seulement l'homme

" réfléchit les perceptions sensibles qu'il reçoit d'une façon qui lui est propre, [mais] il progresse des simples impressions aux représentations, à la différenciation, à la comparaison, au jugement [...]. En lui seul, la somme des sensations est unie à une totalité qui a sa place, son organe, son existence spécifique dans la force unificatrice, dans le moi $» 6$.

C'est dans la mesure où il y a adéquation entre les « catégories intellectuelles et morales de celui qui considère et celles qui s'expriment dans ce qui est à interpréter " 7 que l'interprétation est possible. Il y a cercle entre le même et l'autre à partir de la notion de similitude : au sein de la finitude, l'homme se construit à chaque fois en tant que totalité et conscience, chaque individualité

" est une particularité grandie à partir des mêmes conditions et activités, chacune avec [son] propre monde et [sa] propre histoire ; chacune est une étrangère pour l'autre et cependant semblable à elle, un morceau du monde extérieur et en même temps un miroir de son propre monde le plus intérieur, compréhensible pour l'autre ${ }^{8} 8$.

C'est parce qu'il est monde, totalité, et qu'il le sait que l'individu peut être compris, totalité signifiant forme délimitée par rapport à autrui, mais ouverte sur autrui car en devenir.

4. Ibid., p. 268.

5. Ibid., p. 413.

6. Johann Gustav Droysen, Historik, Textausgabe von Peter Leyh, Stuttgart/BadCannstatt, Fromman-Holzboog, 1977, p. 23 (cité par la suite comme DroYSEN).

7. Ibid., p. 22.

8. Ibid., p. 24. 


\section{1. Image totalisante et identifiante}

Le rapport entre l'historien et son objet est un rapport spéculaire. Le rôle de la vision est essentiel chez Ranke alors que chez Droysen, l'interaction se fait dans et par le langage.

Chez Ranke, l'histoire doit reproduire, refléter les phénomènes du passé : « lorsque la vie se reflète pour l'homme dans l'esprit concevant, se fixe par l'observation, elle est préservée de la ruine et acquiert une immortalité terrestre ${ }^{9}{ }^{9}$. " L'esprit humain fait face à lui-même et se reconnaît, c'est pourquoi l'histoire est immédiatement la vie de l'esprit humain $"{ }^{10}$ : le miroir est le lieu de la reconnaissance de soi dans l'image reflétée, dans sa propre image, et des autres considérés comme semblables, d'autant plus semblables que l'époque étudiée se rapproche de celle du chercheur. Par l'image, l'historien (ou le genre humain) prend possession de son passé, prend conscience de lui-même dans sa propre totalité : « le genre humain devient conscient en tant qu'unité en premier lieu par l'histoire" " che historique est la mise en évidence du moment dans lequel le genre humain prend conscience de son unité.

Chez Droysen, il s'agit de comprendre « le langage des temps passés ». La langue est l'expression immédiate de l'intériorité, " une de ces extériorités dans laquelle la totalité la plus intérieure de l'homme jaillit " ${ }^{12}$. Elle est la médiation entre notre conscience et celle d'autrui, ce qui est le plus individuel et particulier et en même temps commun et général. L'histoire est science de l'intériorité : la compréhension se fait au niveau de l'âme :

" chaque mouvement de l'âme le plus intérieur de l'un est tout de suite accessible à l'autre en perception sensible [...]. À partir de la même disposition en lui-même, il peut concevoir ce qu'il perçoit en donnant en retour une signification ${ }^{13}$.

Nous avons ici des éléments proches des catégories lacaniennes d'imaginaire et de symbolique - élargissement de la notion hégélienne d'aliénation - " " L'œil est fondamental dans l'imaginaire lequel renvoie au rapport du sujet avec ses identifications formatrices et avec le réel » : la collectivité, par ses historiens, forme son identité, s'unifie

9. DotTerweich, Fuchs, p. 119.

10. Ibid.

11. Ibid., p. 140.

12. DRoYsEn, p. 26.

13. Ibid., p. 25. 
en tant que telle. Chez Ranke, il y a une incorporation de l'objet historique en une identification immédiate et directe, en une fusion narcissique. L'historien tend à prendre son point de vue au-dessus du présent. L'histoire évolue ainsi dans un " présent éternel ", elle est la vie de l'esprit humain reflétée dans une image fixe. Mais « cette identification assure l'avènement d'un sujet par le fait que l'image réfléchie de soi-même est une aliénation fondamentale ". Ceci est particulièrement net chez Droysen où chaque individualité est " décalée " par rapport à elle-même, où le langage est ce qui singularise au moment même qu'il unit. L'imitation, la mimesis, à l'origine du langage (comme de l'activité artistique proche de l'activité de l'historien) n'est pas la simple répétition d'un monde extérieur mais un projet créateur : la reproduction de l'objet est la saisie de ses rapports de structure, lesquels ne peuvent être compris que lorsqu'ils sont construits. Ainsi le présent reçoit son identité par le passé, mais par la façon dont il actualise le passé. Ainsi Droysen dit que l'historien scrute, domine sciemment, volontairement le monde des phénomènes.

\section{2. Dieu ou la totalisation absolue}

La totalisation est circularité entre moi et autrui, séparation et recherche du complément, recherche d'un être absolu à qui est transférée la qualité de l'ubiquité : Dieu. Le passé est la projection idéalisée de la collectivité. L'histoire aspire continuellement à atteindre son modèle. Elle conduit à la "vérité " de l'existence humaine en faisant connaître la nature spirituelle dans l'aspiration continue de l'humanité vers le perfectionnement. Chez Ranke, la série de ces aspirations élève l'aspiration de l'historien, rend son regard moins partisan :

« [l'histoire] devrait embrasser l'extérieur, révéler l'intérieur, car sans ceci, cela serait seulement l'enveloppe, sans noyau, et rendre présent à l'esprit les deux, en même temps. On n'objecte pas qu'elle serait elle-même une partie du savoir divin auquel nous aspirons ; le savoir divin est la vérité à laquelle aspire toute science $» 14$.

L'historien aspire à l'ubiquité mais sait qu'elle est l'affaire de Dieu :

« il est impossible, comme dans toute autre science, d'embrasser [le passé] totalement ; car même la connaissance la plus étendue ne pourrait jamais être à la disposition, en un instant, de ce qui est unique, car le plein de la vision ne peut appartenir qu'à Dieu » 15 .

14. Dotterweich, Fuchs, p. 199.

15. Ibid., p. 120. 
Pour Droysen, plus l'homme s'élève, plus s'élargit l'horizon qu'il domine du regard :

" la vérité absolue est comme le soleil des soleils la source de la lumière qui éclaire jusqu'aux dernières lunes, détermine de même et porte le mouvement.

[Ces] cercles, si nous les suivions jusqu'à leur noyau, nous montreraient l'image du monde moral comme il apparaît à l'œil de Dieu, son étant devenu comme un présent éternel [...]. Celui qui a parcouru ces cercles s'apercevra, en pensant historiquement $[\ldots]$, que leur noyau est à nouveau dans la périphérie d'un cercle encore plus haut " 16 .

La représentation a une source solaire, elle est lumière qui fait voir et à laquelle l'homme aspire à s'égaler de perfectionnement en perfectionnement. L'unité idéale est à l'intérieur du moi illuminé par elle : le tout est l'intériorité de la conscience. L'esprit fini a seul là capacité en se souvenant et espérant, de donner à l'instant fluctuant une manière qui est une copie de l'éternité de Dieu : " par l'histoire nous comprenons Dieu, et seulement en Dieu nous pouvons comprendre l'histoire " ${ }^{17}$. Droysen nomme l'histoire une théodicée, la vision éthique du monde tendant à ce que tout vouloir se comporte vis-à-vis de Dieu en plaçant en lui son élargissement vers la totalité et sa justification.

\section{3. Le système de la connaissance historique}

À partir de ce fondement spéculaire et intersubjectif, la recherche historique apparait dans l'historisme comme un ensemble de processus articulé par l'" interprétation ". Chez Ranke, le système du savoir historique - conduisant à la connaissance pure - comprend deux ou trois éléments de dénomination et de combinaison variables. L'auteur parle par ordre dégressif de critique, de vision et de compréhension. Ainsi :

« la tâche du véritable historien consiste :

1) dans la critique : l'examen de ce qui est recherché est l'exigence principale pour une compréhension exacte.

2) À partir de là, la compréhension se donne comme une condition très importante. L'observation des différents rapports, de la représentation et du mode de pensée des gens, quelles forces ceux-ci mirent pour atteindre cela, ce qui arriva.

3) Synthèse : la vie générale du genre humain doit être saisie. La critique

16. DROYSEN, p. 61-62.

17. Ibid., p. 30. 
donne en conséquence le sol solide, la compréhension de la connexion lui fait suite ${ }^{18}$.

La vision est globale, l'historien considère (betrachtet), observe (beobachtet). La perspective historique a pour préalable une élévation spirituelle vers le lieu de la magnificence de l'universel. L'élévation du regard est en même temps accès à la pureté et à la capacité englobante, il ne doit pas être limité par l'esprit de parti. La compréhension est présentée comme complémentaire : 1) de la vision : « [l'histoire] est d'après son essence vision et compréhension " ${ }^{19} ; 2$ ) de la recherche de l'objectivité des faits : " le principe de la recherche historique [...] est dirigé vers la discussion objective des faits et leur compréhension spirituelle $"{ }^{20} ; 3$ ) à la fois de la vue et de la combinaison : " la vue, l'assemblage, le fait d'apprendre à comprendre les monuments transmis par l'écrit sont l'affaire de l'historien " ${ }^{21}$; 4) de la critique et de la synthèse : la compréhension de la connexion des modes de pensée du passé est pensée relationnelle et non intuition pure. La connaissance historique est apprẻhendée comme exactitude. Le système de la connaissance chez Droysen comprend l'heuristique, la critique et l'interprétation. Le point de départ est la "question historique »: le chercheur pose une question déterminée et son souci est d'obtenir la réponse à sa question, grâce au matériau. L'acte de la recherche s'inscrit dans la totalité de la continuité en perpétuant le souvenir par la recréation de la tradition. Il dépend de la culture (Bildung) de l'historien, c'est-à-dire de l'appropriation du passé par la conscience : « avoir retravaillé, avoir revécu en esprit conformément à la pensée, comme une continuité ce qui a été élaboré dans l'histoire des temps, des peuples, de l'humanité, cela s'appelle culture " ${ }^{22}$. La critique montre que l' " état des choses " fourni par le matériau assemblé par l'heuristique est en rapport avec les « actes du vouloir " des individus. Elle comprend : 1) la critique de l'authenticité : il s'agit de savoir si les matériaux sont le réel pour lequel ils sont tenus ; 2) le procédé diacritique, à savoir si les matériaux sont encore ce qu'ils étaient à l'origine ; 3) la critique de la justesse qui recherche si, à cause de ce devenir, les matériaux sont relativement vrais ; 4) le classement critique qui considère jusqu'à quel point les matériaux sont incomplets. À partir de là, l'historien a « à comprendre en cherchant »

18. DOTTERWEich, Fuchs, p. 157.

19. Ibid., p. 86.

20. Ibid., p. 412.

21. Ibid., p. 203.

22. DROYSEN, p. 71. 
et non à expliquer. Il n'explique pas ce qui est devenu comme un résultat nécessaire des conditions historiques, mais interprète, signifie une individualité par la totalité de ses moments. L'interprétation est un ensemble de quatre éléments : 1) l'interprétation pragmatique qui saisit les données dans leur objectivité (Ranke serait resté à ce niveau, au niveau de l'exactitude) ; 2) l'interprétation des conditions qui pense que les conditions étaient contenues idéalement dans la réalité d'un moment ; 3) l'interprétation psychologique qui étudie les motivations des participants à l'action ; 4) l'interprétation des forces morales ou idées : « les choses vont leur chemin en dépit de la bonne ou de la mauvaise volonté de ceux par qui elles s'accomplissent $"{ }^{23}$.

\section{L'OBJET HISTORIQUE : UNE GLOBALITÉ}

\section{1. Imbrication monde/État/nation/peuple}

L'histoire est une pensée de la concentration au sein de l'État-nation. L'objet primordial est l'État, complexe plus ou moins large. L'État (" forme ", " organisation ") coïncide le plus souvent avec le peuple et la nation, ces deux termes étant équivalents, et fait partie d'une synthèse plus grande, l'histoire universelle. Le niveau scientifique apparaît alors comme un reflet du niveau socio-politique, du moment de l'unité nationale allemande.

Ranke est l'historien de l'Universalgeschichte : l'histoire de l'humanité est un " tout étroitement uni ${ }^{24}$. Elle n'est pas une simple histoire diplomatique mais elle met en évidence ce qui relie les peuples et constitue l'unité du genre humain au-delà de toute différence. La totalité objective est soit la vie des personnalités éminentes, soit le peuple, soit l'« atmosphère spirituelle " d'une époque dont les éléments dynamiques sont l'État, l'Église et la littérature :

" il y a dans le monde des puissances invisibles, lesquelles sont les plus efficaces de toutes et constituent la totalité [...]. L'État, à qui chacun appartient, les convictions religieuses qui s'emparent de l'individu, les grandes différences qui débouchent sur l'État et l'Église, et fermentent les esprits éternellement, forment une atmosphère spirituelle dans laquelle on respire et vit $" 25$.

23. J. G. Droysen, Précis de la science historique, trad. par P.-A. Dormoy, à partir

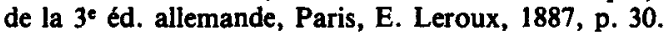

24. DOTTERWEich, Fuchs, p. 405.

25. Ibid., p. 413-414. 
De même,

« la totalité existe finalement. Si c'est une vie, nous saisissons son apparition ; nous prenons comme vraie la succession, la relativité d'un moment à travers l'autre [...] Ce qui est total est aussi certain que chaque extériorité dans chaque moment. Si c'est un peuple, alors ce n'est pas seulement [dans] les moments individuels de son extériorité animée, mais à partir du tout de son évolution, de ses actes, de ses institutions, de sa littérature, que nous parle l'idée 》" 26.

Peuple et nation sont équivalents. L'interaction des peuples au sein de l'histoire universelle (cette dernière étant limitée aux nations occidentales) repose sur la liberté. Il importe " qu'un peuple reste libre et développe les éléments étrangers librement. Un peuple opprimé n'apporte qu'une culture secondaire " ${ }^{27}$. L'État repose sur le patriotisme : " la politique intérieure doit être dirigée vers la synthèse de toutes les parties en une unité de libre vouloir $"{ }^{28}$. Les instruments en sont la bienveillance, le bien-être général, l'information. L'autonomie par rapport à l'extérieur donne à l'État une signification universelle : elle est permise si l'État est une " grande puissance ».

Chez Droysen, le peuple, " produit historique ", est primordial. Il est à la fois le cadre et la possibilité de la cohésion par son identité morale. Si bien qu'il fait, de ce qui est devenu historiquement, la nature innée du genre humain :

« chacun ressent qu'il a dans son mode populaire [Volksart] ce qui est le plus saint, sa morale naturelle, et il a le droit et le devoir d'y tenir ferme avec toutes ses forces et de prendre fait et cause pour lui $\gg 29$.

La conception de l'État est ambiguë. Ce dernier est à la fois un État de droit et une forme totalisante. Puissance publique organisée, il est seulement une catégorie parmi d'autres dans lesquelles la communauté du peuple peut apparaître. Il est le lieu de l'interaction dynamique et libre des autres « communautés morales » (totalités relatives englobant le moi de la personnalité), il ne les protège pas seulement, il les représente. Le modèle en est l'État prussien de Stein. Stein opéra un " rapprochement " entre la force politique et la liberté civile, augmenta la force du peuple.

26. Ibid., p. 82-83.

27. Ibid., p. 138.

28. Leopold von RANKE, Politisches Gespräch, Göttingen, Vandenhoeck und Ruprecht, 1963 , p. 67.

29. DROYSEN, p. 307. 
La réforme politique ne prend pas sa source dans le principe du droit divin du pouvoir légitime, ni dans celui de la souveraineté du peuple, ni dans le droit historique, mais dans les idées nationales : l'État appartient au peuple et réciproquement. La nature morale de l'État se fonde sur la force de la dignité humaine, l'amour de Dieu, du roi et de la patrie. L'État consiste en une série de systèmes représentatifs existant les uns par les autres, fondés sur les communautés urbaines et rurales. L'organisation militaire est une partie essentielle de la liberté politique ; elle arme le peuple comme peuple : la nation vise ainsi non pas à former une puissance mais bien un État. Droysen projette un système confédéral des États embrassant la terre :

" au sein du chaos ethnographique, il se dégage comme pure cristallisation État sur État. De la maxime adversus hostem aeterna auctoritas esto, leurs relations mutuelles marchent progressivement jusqu'au régime des traités et du commerce pacifique, jusqu'au droit des gens. État de confédérés, confédération d'États, système d'États, système d'États universels, tels sont les cercles concentriques de ce mouvement qui va toujours s'élargissant $" 30$.

Le développement de l'histoire va de pair avec celui de la pensée unitaire.

Chez Lamprecht, l'évolution " historico-mondiale » est articulée sur le développement des « âges culturels " (Kulturzeitalter) des nations, sur la totalité de leur vie psychique (principalement) et matérielle : « la vie de l'âme d'une époque déterminée forme en soi un tout, une unité, dont les effets de la moindre activité humaine ne peuvent être éloignés sans lui prendre son caractère " ${ }^{31}$. Nation et État sont considérés par rapport à la trilogie nature-société (nombre)-culture. La nation, « somme des formations de groupe " est une " formation socio-naturelle ". Plus elle est naturelle et plus elle est sociale ; l'État " est cette organisation sociale à laquelle toutes les autres se subordonnent $[\ldots]$; il est la plus haute réunion sociale à cause de la culture $"{ }^{32}$. La nation est primordiale :

« porteuses régulières de l'évolution historico-mondiale et par conséquent fondement le plus important de l'histoire de façon générale, [telles] sont [...]

30. J. G. DROYSEN, op. cit. supra n. 23, p. 42.

31. Karl LAMPRECHT, Einführung in das historische Denken, Leipzig, Veröffentlichungen der Gesellschaft Neue Bahnen, Voigtländer, 1912, p. 65.

32. ID., "Was ist Kulturgeschichte ? Ein Beitrag zu einer empirischen Historik », Deutsche Zeitschrift für Geschichtswissenschaft, 1896-1897, réimpr. in K. LAMPRECHT, Ausgewählte Schriften zur Wirtschafts- und Kulturgeschichte und zur Theorie der Geschichtswissenschaft, Aalen, Scientia Verlag, 1974, p. 281. 
les nations : le plus grand type d'évolution de l'existence humaine s'achève seulement en elles. Cette existence apparaîtra d'autant plus unie que la nation est en même temps fondement d'un État; cependant cette liaison avec un corps étatique uni n'est pas nécessaire " ${ }^{33}$.

Chez les trois historiens, le patriotisme permet l'unité globale : l'histoire contient une théorie de la construction culturelle volontaire reposant sur le consensus gentium. État et nation sont les objets les plus marquants. Or Droysen et Lamprecht montrent le passage vers une complexité plus grande et une socialisation de l'histoire. Ainsi l'État est seulement une " communauté morale " (Droysen) ou une " force psycho-sociale » (Lamprecht) parmi d'autres.

\section{2. Des communautés morales aux forces psycho-sociales}

Chez Droysen, l'objet historique est une imbrication de configurations : "les formes dans lesquelles le travail de l'histoire se meut sont les communautés morales dont les types se trouvent comme forces morales dans le cœur et la conscience des hommes $"{ }^{34}$. L'auteur différencie trois communautés : les communautés naturelles (la famille, les lignées, le peuple), les communautés pratiques ou intermédiaires (la société, le bien-être, le droit, l'État), les communautés idéales (la langue, l'art, les sciences, la religion). Les systèmes que forment les communautés morales sont en mouvement les uns vers les autres. Chaque système a une qualité relative. Deux directions sont primordiales : l'équilibre (il y a excédent ou relativité dans l'ensemble) et la totalité (les communautés ont en commun le " besoin de se compléter jusqu'à la totalité »). Ainsi l'élément se justifie dans et par la totalité : la famille tend vers le peuple, l'idée de peuple s'accomplit dans l'idée d'humanité,

" c'est-à-dire la connaissance qu'il y a au-dessus des particularités naturelles par lesquelles le monde humain est différencié, l'unité de la nature spirituelle $"{ }^{33}$.

L'humanité se réalise dans la Chrétienté. Les idées de bien-être et de droit atteignent leur équilibre dans la sphère de l'État mais freinent l'idée de puissance de ce dernier. L'art et la science se complètent dans la religion. Les communautés morales se différencient en fonction de leur relative naturalité ou relative idéalité et de leur degré de liberté. Les commu-

33. Ibid., p. 284.

34. J. G. DroYsen, op. cit. supra n. 23, p. 36.

35. DroYsen, p. 308. 
nautés naturelles tendent à devenir historiques. Elles sont le lieu du renouvellement de l'espèce dans l'individu. Leur dimension corporelle les rend substantielles (elles " changent en se conservant "). La personnalité y est déterminée, la liberté y est « liée ». Les communautés idéales sont le lieu de l'acquisition culturelle et de l'activité spirituelle (elles " se conservent en changeant "), elles aspirent à devenir « corporelles " dans des institutions. La personnalité n'y est pas déterminée mais s'adonne à l'idée. La liberté tend à se concrétiser au sein de limites données. Les communautés pratiques sont le lieu de l'interaction du corporel et du spirituel, du " combat constant ", de l'identité contradictoire qui aspire à la conciliation, à l'équilibre. La personnalité, forme de l'idée historique, est à la fois déterminante et déterminée ; la limite joue son rôle : elle est la reconnaissance de la liberté de l'autre.

Pour Lamprecht, la masse et non l'individu est l'objet de l'histoire. Les données historiques forment une "série libre-individualiste " et une "série nécessaire-collectiviste "; cette dernière comprend les facteurs naturels et les "facteurs culturels c'est-à-dire psychosociaux ", ces derniers seuls ont une valęur causale. Ils se présentent comme une imbrication logico-chronologique. Les plus primitifs

« sont, au sein de la vie instinctive, l'aspiration vers la conservation de l'espèce ; au sein de la vie de la sensation, le besoin de l'élévation et de la progression des impressions ; au sein de la connaissance finalement, l'usage lié à la langue des formes de pensée les plus simples $"{ }^{36}$.

Le processus psychique le plus simple est celui de la sensation ; s'y réduisent le vouloir, la représentation et le sentiment qui donnent respectivement l'économie, le langage et la " direction vers une élévation des fonctions de l'existence $"{ }^{37}$, l'art. Puis la représentation crée les formes mythologiques, le vouloir crée les mœurs, l'élévation de la sensation crée d'autres formes d'art. La liaison de ces trois éléments donne lieu à quelque chose de qualitativement nouveau, la religion. Alors se développe à partir des mours, dans la genèse de l'État, le droit ;à partir du mythe, alors que la paix demande une plus haute spiritualité, la science ; à partir d'une plus haute vie de la sensation, l'art plastique et la rhétorique.

36. K. LAMPRECHT, op. cit. supra n. 32, p. 300.

37. Ibid., p. 303. 


\section{DE LA CONNEXION A LA SITUATION : L'HISTOIRE, UNE MORPHOGENESE}

La méthodologie pose la question de l'ordonnancement des fragments transmis. Cet ordonnancement est unification, structuration de l'objet, disposition à la fois spatiale et temporelle. La continuité manifeste le changement des formes de l'objet. Elle est progression chez Droysen et Lamprecht.

\section{1. Connexion (Zusammenhang) et moment historico-universel chez Ranke}

L'historisme prône la singularité de l'objet mais cette unicité est le lieu même de l'efficience de la totalité. L'historien a pour fonction de montrer l'interaction entre le particulier et le général. La totalité est l'« intériorité " de l'objet, son " essence spirituelle ». Ainsi Ranke dit que l'idée générale constitue la particularité du phénomène individuel :

« il est nécessaire de s'emparer de l'individuel. Ce n'est que lorsque nous faisons en sorte d'apprendre à connaître, voir et pénétrer les événements individuels que nous pouvons espérer percevoir les idées dans leur plénitude. Ce n'est que lorsque nous nous élevons, d'un côté, à la vision du tout, que nous pouvons espérer saisir l'individuel qui, dans son principe de vie a part à la vie de la totalité $" 38$.

\section{Le phénomène historique a pour nom événement :}

« le fait n'est pas la frontière extérieure ; ce qui devient n'est pas la dernière chose que nous avons à connaître. Il y a quelque chose qui s'est passé là-dedans. L'événement résulte d'une série de faits combince spirituellement.

Notre tâche est alors de reconnaître ce qui, dans la série des faits, s'est intrinsèquement passé $\gg 39$.

La série est le lieu du lien des phénomènes : « une série d'événements - l'un après l'autre, l'un à côté de l'autre - liés ensemble de telle façon, forme un siècle, une époque " 40 . Ce qui s'est " intrinstquement passé 》 est la réalité historique. L'événement est spiritualité (« [l'esprit] emplit toutes les limites de son existence avec son présent ; rien n'est accidentel en lui, son apparition est fondée dans le tout " ${ }^{41}$ ) et combinaison, " liaison interne " (innerliche Verbindung) ou " connexion ». La notion de connexion traduisant véritablement l'unité interne et les rapports avec

38. Dotterweich, Fuchs, p. 202-203.

39. Ibid., p. 177.

40. Ibid., p. 296.

41. Ibid., p. 125 . 
l'extérieur, signifiant à la fois l'ensemble et les éléments de la complexité, le système et ses éléments, fait de l'histoire la science du système. Cette notion se retrouvera dans toute l'historiographie allemande et chez Lucien Febvre. La connexion se manifeste dans l'évolution causale :

" [nous ne disons pas] qu'il y a entre les événements différents seulement une succession. Il y a entre eux une connexion. Le simultané a contact avec l'un et agit sur l'autre. L'antécédent conditionne ce qui suit ; c'est une liaison interne de causes et d'effets $\$ 42$.

La connexion est le fondement du phénomène et sa signification. Elle est l'esprit, l'ensemble structuré qui se présente comme "comment ". L'historien a à fonder la signification interne du phénomène lorsque ce dernier est apparu dans la visibilité.

L'historien considère les systèmes du passé ou " esprit(s) du temps ", " mode(s) de représentation ", " degré(s) de culture ", en tant que lieux de forces en devenir ou " tendances " ou " idées ». Il doit " observer » à la fois le noeud des tendances et chaque tendance en tant que longue série évolutive :

"l'historien a alors à démonter (auseinanderzunehmen) les grandes tendances des siècles et dérouler (aufzurollen) la grande histoire de l'humanité qui, précisément, est le complexe (Komplex) de ces différentes tendances " ${ }^{43}$.

L'histoire est morphogénétique, la désorganisation du système permet le mouvement : " c'est un état de désorganisation qui se manifeste de cette façon. C'est un organisme venu en dispute avec lui-meme " 4 ; la transformation provient de la modification (Modifikation) des éléments. Le lieu de la transformation est le moment historico-universel ou changement d'une forme spirituelle : il joint l'évolution et la contemporaneité du moment, il est le lieu de la connexion générale ; il

" ne reside pas seulement dans la combinaison de ce qui se suit dans les siècles, du contenu, ainsi, des époques différentes, mais aussi dans la jonction [Verknüpfung] du simultané, dans l'interaction [...] du complexe des phénomènes politiques et religieux, artistiques, sociaux, avant tout de la réaction des différentes nations $"$ " 3 .

42. Ibid., p. 79.

43. Theodor SCHIEDER, Helmut BERDING, eds, Leopold von Ranke. Uber die Epochen der neueren Geschichte, Munich/Vienne, Oldenbourg, 1971, passage sténographie, p. 67.

44. DOTTERWEICH, FUCHS, p. 82.

45. Ibid., p. 415. 
Il n'y a pas de progrès, " chaque époque est immédiate à Dieu ".

\section{2. Idée et mouvement chez Droysen}

L'Ideengeschichte de Droysen présente le début d'une pensée de la situation. L'histoire de l'humanité se meut dans les idées et réciproquement. Celles-ci sont des " forces instinctives ", des "forces morales " en devenir, relativement absolues :

" les idées éthiques et l'idéal sont les contre-images idéales de ce que nous avons et connaissons dans ce qui est devenu ; elles sont la formation complétée jusqu'à la totalité de ce qui semble dans la réalité seulement approximatif, seulement successif, et toujours de façon encore incomplète, encore en devenir ${ }^{46}$.

Chacun vit dans le " cercle des idées » de son présent. Les idées aspirent à se réaliser dans l'histoire, donnant forme au moi de l'être humain. L'expression est permise par le jeu de l'idée face à la « situation ".

La situation apparaît comme une complexité contraignante. C'est ce qui pousse les hommes à être conformes:

" chaque moi pense ainsi, [...] agit ainsi, est contraint par la morale, le droit, l'habitude de penser, d'agir ainsi. Combien sont ainsi au sein des situations [historiques] parce qu'on aurait fait ainsi, cru ainsi autrefois " ${ }^{47}$.

L'âme individuelle (niveau auquel aspire à accéder l'historien) se tend dans la polarité de ses côtés " paresseux » et " actif ", là-bas ce à quoi elle est habitué, ici ce qu'elle espère, là-bas la somme du passé, ici le noyau du futur :

" dans la mouvance vivante du présent, quand la vie historique donne d'autres pulsations, se développe toujours de nouveau l'opposition de ce qui est et de ce qui doit être, de la situation et de l'idéal " 48 .

La totalité est dynamique, n'est pas formée, fermée une fois pour toutes, va du centre à la périphérie de son cercle, est la dialectique entre mouvement et unité de l'esprit, entre infini et finitude :

" le mouvement continu de l'esprit se repose seulement dans le concept du tout ; comme il est totalité lui-même, il ne peut se reposer tant qu'il

46. DROYSEN, p. 39.

47. Ibid., p. 204.

48. Ibid., p. 377. 
n'a pas traduit à partir de ce qui le meut en pensant, l'image d'une totalité $\${ }^{49}$.

L'histoire est le lieu des changements qualitatifs des formes de la pensée : le genre humain est en progrès continuel. La progression est permise par le décalage entre l'idée et son expression :

"l'insuffisance des phénomènes individuels se complète dans [les] idées ; nous reconnaissons que l'expression de ces idées dans le phénomène individuel n'est jamais adéquate et finale ; la continuité et le mouvement expriment globalement et approximativement l'idée [...]. Ce qui est le plus proche de la perfection est le progrès [...]. L'histoire est le devenir progressif et la croissance de l'idée " $\$$.

\section{L'histoire est téléologie.}

\section{3. Situation et intensité progressive chez Lamprecht}

« Les situations ne sont pas de la matière mais au contraire des complexes d'effets psychiques massifs, des forces psycho-sociales ; et elles se transforment avec le changement psychique de ces complexes " 51 .

La psychohistoire de Lamprecht considère la situation - ensemble de processus communs psychiques en devenir - comme causalité. L'auteur insiste sur la notion d'une structure légale, non statique, des phénomènes historiques et, par ailleurs, sur la possibilité de l'existence, dans cette structure, d'un éément qualitatif et créateur, sur la possibilité d'une spécificité au-delà de la nécessité ; la caractéristique de l'unité de la structure est exprimée par la "loi des relations psychiques » : une structure se manifeste lorsque se mettent en place, en fonction d'une transformation des éléments les plus récents, des interrelations; la nouveauté d'un seul elément peut agir sur la totalité structurelle et inversement :

" [il s'agit de] la loi d'aprés laquelle la situation globale [Gesamtzustand] de la vie de l'âme se met en place d'apres les influences les plus jeunes qu'elle expérimente. Et on peut encore une fois genéraliser cette loi des relations psychiques en l'élargissant à tout organisme. Toutes les parties se condi-

49. Ibid., p. 31.

50. Ibid., p. 201.

51. K. LAMPRECHT, "Der Ausgang des geschichtswissenschaftlichen Kampfes", Zukunft, 20, 1897, p. 201. 
tionnent tout à fait dans une constellation de sorte que la dernière transformation d'une partie résonne dans la transformation analogue de toutes les autres $[. .$.$] .$

Où cesse finalement l'application du concept d'organisme ? Et à qui n'apparaîtrait pas sur le chemin où nous marchons depuis longtemps un dernier but visiblement supérieur, la loi de l'équilibre des énergies ? " $\mathbf{5 2}$.

La nouveauté structurelle de la situation a un aspect qualitatif exprimé par la « loi des résultantes historiques », application du " principe de la synthèse créatrice " de Wundt sur les conditions particulières de l'évolution historique. Le lieu où fonctionne la résultante est l'unicité historique, ensemble qui n'est pas l'a priori d'une synthèse de plusieurs conditions historiques, mais est nouveau dans et par son unicité. En tant que totalité, il est plus que les éléments qui le composent, éléments agissant cependant comme répercussion qualitative de chaque condition. Ce n'est que lorsqu'on fait entrer une dimension valorisante que la suite des conditions est considérée comme allant vers un but déterminé :

« chaque unicité est l'effet résultant d'une pluralité de conditions historiques avec lesquelles elle est en rapport - de sorte qu'en elle la nature qualitative de chaque condition a des répercussions -, et possède un caractère à la fois nouveau et unitaire qui dérive dans l'analyse historique de la liaison de ces facteurs historiques, mais à partir desquels on ne peut jamais construire une synthèse développé a priori. Un cas particulier se produit quand la liaison causale des événements historiques ne peut jamais être exposée avec sûreté par un procédé progressif, comme cela a coutume d'arriver dans le domaine des sciences de la nature, mais seulement par une conclusion de l'action. Mais assurément aucune caractéristique téléologique n'est liée en soi avec cela [...]. Cela arrive avec la réception éventuelle d'une détermination de valeur, car le but est seulement cette suite de conditions antérieures à laquelle est attribuée une valeur afin que cette unité précisément soit considéréé, à cause de cette valeur, comme un but. Mais certainement, la loi des résultantes historiques est aussi particulièrement valable pour les liaisons de causalité subjectives inversées, c'est-a-dire les liaisons de but $[\ldots .$.$] et pour les buts particulièrement éminents historique-$ ment. Cette transformation téléologique, Wundt l'a formulée plus tốt de façon particulièrement aiguë comme "principe de l'hétérogéneité des buts" " 33 .

Dans les "phénomènes historiques massifs ", c'est-à-dire les sentiments, les volontés, les représentations communs, cette dimension

52. ID., "Über den Begriff der Geschichte und über historische und psychologische Gesetze ", Annalen der Naturphilosophie, 1903, p. 269.

53. ID., art. cit. supra n. 32, p. 276-277. 
qualitative est le fait d'un moment psychique particulier, l'« accord social ", ou « habitus global spirituel ", " comportement psycho-social ", " psyché sociale ", " diapason psychique »... Cet accord social, " conséquence de la transformation des conditions suprêmes, c'est-à-dire des degrés typiques du devenir national " $^{54}$, est un consensus. La causalité particulière de la synthèse créatrice est liée à un " excédent " en forces psychiques. Ce dernier a une dimension évolutive, progressive en tant qu'intensité progressive d'un âge culturel à un autre, et implique la régularité de l'évolution vers une accession plus grande de l'âme à la conscience. Les étapes de l'évolution empiètent l'une sur l'autre. Les facteurs psycho-sociaux les plus anciens embrassent tous les corps culturels, même s'ils agissent de façon plus rudimentaire dans les âges culturels plus tardifs. La signification de ces facteurs s'efface de plus en plus non parce qu'elle devient en elle-même plus faible mais parce qu'elle est recouverte par celle des facteurs spécifiquement spirituels. L'" activité d'imagination " (Phantasiethätigkeit) est le modèle scientifique parfait ; c'est la « fonction psychique au sein de laquelle la création combinatrice de nouvelles unités à partir d'éléments donnés - ainsi le processus authentique de l'analyse [...] et de la synthèse s'accomplit le plus facilement " ${ }^{35}$. Les rapports d'unité entre les individus et les situations au sein de l'âge culturel sont plus profonds dans les "étapes culturelles inférieures " parce que l'individuation des individus a moins progressé au sein de la différenciation encore minime de la culture globale.

\section{CONCLUSION : DEUX NOUVELLES HISTOIRES ? \\ K. LAMPRECHT ET LES ANNALES}

La méconnaissance en France des cuvres de Ranke, Droysen, et particulièrement Lamprecht, pose le problème de l'histoire de l'histoire, savoir en construction. L'histoire de l'histoire a à surmonter l'ecueil du gallocentrisme qui présente, en une marche triomphante, la nouveauté de l'historiographie des Annales par rapport à l'histoire événementielle, " vouée au récitatif politique et biographique » de l'histoire positiviste. Ce dernier terme « recouvre par excellence la manière dont il ne faut pas,

54. ID., " Individualität, Idee und sozialpsychische Kraft in der Geschichte ", Jahrbücher für Nationalökonomie und Statistik, 68, 1987, p. 886.

55. ID., Moderne Geschichtswissenschaft, Fünf Vorträge, Freiburg i. Br., H. Heyfelder, 1905 , p. 122. 
ou plus faire de l'histoire " ${ }^{56}$. Que fait-on de la coupure épistémologique instaurée par Lamprecht ?

L'historiographie allemande aurait succombé à la philosophie. On ne retient de l'Allemagne que les constructions de la philosophie de l'histoire...

Or il ne s'agit pas d'énumérer des conquêtes au niveau de l'élargissement du champ historique, ni d'étudier des thèmes historiographiques, mais d'analyser les concepts inherents à l'histoire, dans leurs parcours et temporalité propres, dans leur articulation. Il s'agit de donner à l'histoire de l'histoire une dimension internationale, traversant les particularités culturelles, et ceci depuis le XviIl' siècle, Voltaire et la Kulturgeschichte.

Le temps de la logique d'une science n'est pas le même que celui de sa sociologie. Ranke a souligné, malgré son orientation individualiste et politisante, que l'histoire était science des relations. Mais l'œuvre de Lamprecht marque une coupure. Elle a été combattue par les historiens académiques officiels de Berlin, attachés à la raison d'État, introduite, par contre, en France par Henri Pirenne et l'histoire synthétique d'Henri Berr, écartée finalement à cause de son orientation völkisch, par Marc Bloch ${ }^{57}$. Or la neue Geschichte fondée sur une methode statistique, étudiant un objet global et régulier, a eu un impact de longue durée. Le lien inconscient, signifié par la notion d'histoire totale, entre les deux mémoires culturelles, est celui d'une époque de la recherche historique, celle des deux premières générations des Annales, alors que maintenant, les historiens - de l'anthropologie historique à l'histoire de l'imaginaire - travaillent plus sur les limites.

La Nouvelle Histoire apparaît, à bien des égards, comme une concrétisation du programme établi par l'ethnologie historique de Lamprecht. Mais elle est plus. Elle est caractériscé par l'ouverture aux autres sciences humaines. Comment ces sciences s'articulentelles? Dans quelle mesure les concepts propres à chaque discipline sont-ils transférés? La méthode typologique de l'induction statistique comparative, recherchant les régularités d'un objet massif présenté comme « série (Reihe) nécessaire-collectiviste " se poursuit dans l'histoire quantitative puis sérielle. Cette démarche s'appuie sur des sources formant des séries de longue durée et vaut non seulement pour le

56. Roger Chartier, "Histoire positiviste ", in Jacques Le GofF, Roger Chartier, Jacques Revel, dir., La Nouvelle Histoire, Paris, Retz/C.E.P.L. (« Les Encyclopédies du savoir moderne $"), 1978$, p. 460.

57. Cf. Catherine Devulder, "Karl Lamprecht, Kulturgeschichte et histoire totale ", Revue d'Allemagne, oct. déc. 1985. 
niveau économique, mais aussi pour tous les niveaux de l'histoire jusqu'au "troisième niveau » de Pierre Chaunu : elle caractérise " toute approche historique qui construit des séries statistiques à partir de données homogènes les unes aux autres, répétées sur une longue durée, comparables à des intervalles donnés et réguliers " ${ }^{58}$. La massivité (Massenqualität) de l'objet introduit l'étude de la culture populaire et des populations.

Une discipline paraît fondamentale pour l'histoire, aussi bien au $\mathrm{XIX}^{\mathrm{e}}$ siècle qu'au XXe : la psychologie. L'accent mis sur l'idéalité chez Ranke et Droysen, sur la psyché sociale chez Lamprecht, faisant de l'histoire une science de l'âme, l'âme soulignant à la fois le nœud des relations et la dimension psychique, donne une épaisseur à l'histoire de l'histoire des mentalités. La recherche des structures psychiques plus que celle des structures économiques, caractérise la production historiographique française.

Le cadre de la recherche a un aspect global. Il s'agit d'aller « de la cave au grenier " (Michel Vovelle). Dans le cadre de la monographie régionale, principalement, ou locale, l'historien parcourt tous les niveaux de l'activité humaine dans son rapport à la géographie (peu étudiée dans la tradition allemande), de l'économie aux mentalités collectives. Mais c'est justement dans sa conception de l'histoire totale que se marque l'originalité française.

D'une part, la globalité « n'est plus recherchée principalement dans la synthèse, mais dans des objets globalisants ${ }^{59}[\ldots]$ permettant de réaliser une véritable interdisciplinarité à la marge " ${ }^{60}$, et, d'autre part, elle intègre des hétérogénéités temporelles. L'historien considère l'enchevêtrement de " prisons de longue durée " (Fernand Braudel), de "résistances au changement " (Ernest Labrousse), d'« histoire immobile » (Emmanuel Le Roy Ladurie), et de temps mobile des élites, de temps court, de "retour de l'événement" (Pierre Nora). La temporalité différentielle des Annales s'oppose à l'homogénéité de la temporalité continuiste de l'historiographie allemande du XIX' siècle, au présent éternel d'un Ranke ou au progrès de Droysen et Lamprecht.

Le concept de "situation ", insistant sur l'organisation relationnelle des donnees, sous-tendait la Gesamte Geschichtswissenschaft de Lamprecht. Il se poursuit dans la « structure ». Or ce terme, emprunté

58. R. Chartier, " Histoire sérielle ", in J. Le Goff, R. Chartier, J. Revel, dir., op. cit. supra n. 56, p. 508.

59. Ainsi dans l'histoire sociale.

60. J. Le Goff, « Présentation », in J. Le Goff, R. Chartier, J. Revel, dir., op cit. supra n. 56, p. 16. 
aux économistes, est employé d'une façon particulière dans la tradition française. La structure est bien une cohérence - elle insiste sur les interactions au sein d'un ensemble donné -, mais elle est surtout un phénomène temporel, de longue durée, une permanence, une stabilité : alors que les historiens du XIX siècle empruntent le langage philosophique et parlent de nécessité face à la liberté, ne suggèrent-ils pas cette même stabilité ?

Il convient de mesurer ce que cela signifie pour l'histoire : avoir un passé à vocation totalisante.

Catherine DEvULDER, Professeur agrégé d'histoire Lycée de Rueil-Malmaison. 